uptake. During the early lag phase, however, and during the logarithmic phase of multiplication, penicillin has a strong inhibitory effect, and eventually completely stops the oxygen uptake, even in small concentrations $(0 \cdot 04-0 \cdot 1$ units per c.c.). They also demonstrated a strong bactericidal effect in the early lag phase and the logarithmic multiplication phase, but no measurable bactericidal effect during the resting phase. This confirms American work. Chain and Duthie conclude that penicillin can exert a bactericidal effect on Staphylococcus before actual division occurs, but that the organism can undergo at least one division in the presence of penicillin when it is added during the logarithmic phase of multiplication. It appears to interfere with some metabolic function of the early stages of bacterial development. Helvolic acid, the bacteriostatic antibiotic, antagonizes the bacteriostatic and bacteriolytic action of penicillin. Sulphanilamide and sulphamezathine, on the other hand, do not prevent the occurrence of several bacterial divisions and have no antagonistic effect on the bactericidal effect of penicillin on Staphylococcus and Streptococcus; on the contrary, they have a synergistic effect.

Chain and Duthie compare the modes of action of penicillin and the sulphonamides and review some of the literature upon this subject. They conclude that penicillin can kill Staphylococcus in the early lag phase before cell division has occurred, while sulphonamide kills only after several divisions have taken place in its presence. These results may be compared with those obtained by W. S. Miller, C. A. Green and H. Kitchen (Nature, 155, 210, Feb. 17, 1945), who estimated the growth of the Oxford Staphylococcus turbimetrically in the presence of penicillin and sulphonamides and also discuss the effects of these two kinds of substance. They suggest that confusion may arise in discussions of bacteriostatic and bacteriolytic action because too fine a distinction is drawn between these two modes of action. Both penicillin and sulphonamides act by inhibiting cell multiplication; but, while sulphonamides inhibit the growth of almost every kind of cell as well as that of bacteria, there is little evidence that penicillin does this. Additional knowledge of the chemistry of penicillin may reveal that its mode of action is, like that of the sulphonamides, related to its chemical structure. It is unlikely that the mode of action of penicillin is unique; it is more likely that it differs from that of sulphonamides only in degree.

P. Bonét-Maury and R. Pérault (Nature, 155, 701, June 9,1945 ), using the differential photometer at the Radium Institute, Paris, suggest that, whereas the sulphonamide used by them (Dagenan $1162 \mathrm{~F}$ ) does not stop but delays proliferation of Staphylococcus aureus, penicillin stops proliferation almost immediately, even at low concentrations, and that lysis follows. They found, however, that later a second proliferation occurred regularly, followed by a second partial lysis and then a second post-lytic growth. These results recall those obtained by $\mathrm{J}$. W. Bigger (see Nature, 155, 403, March 31, 1945). Bonét-Maury and Pérault obtained their results with six different penicillins (English, American and French). J. Hirsch (C.R. Ann. Soc. Turque des Sci. Phys. et Nat., 12 ; 1943-44) also studied the action of penicillin in vitro by manometric methods, and his work is discussed by Chain and Duthie (loc. cit. above). R. Knox (Lancet, 559, May 5, 1945) also concludes that the action of penicillin can be bacteriostatic, bacteriolytic or bactericidal, and that the young bacterial cell is particularly susceptible to penicillin. A. B. MacGregor and D. A. Long (Nature, 155, 201, Feb. 17, 1945), studying the action of penicillin in relation to their work with penicillin pastilles (see Nature, 341, March 17, 1945), conclude that penicillin is bactericidal as well as bacteriostatic.

Very instructive also is the direct microscopical observation by Prof. A. D. Gardner (Lancet, 658, May 26, 1945), whose earlier work (Nature, 146, 837 ; 1940) suggested that weak penicillin inhibited growth but did not prevent it, on the effects of penicillin on the spores of $B$. anthracis and $B$. subtilis. Gardner found that strong penicillin (5C-100 units per c.c.) slowly kills the spores. Those of $B$. anthracis were more susceptible than those of $B$. subtilis, but neither were completely eliminated and there was no gross swelling. The spores gradually lost their high refractility and became empty ghosts. Weaker solutions of penicillin $(0 \cdot 1-1 \cdot 0$ units per c.c.) allowed the earliest phase of germination to occur, and the spores of $B$. anthracis then swelled up and became spherical coccoids which burst. Those of $B$. subtilis underwent similar changes, but all were not killed. Nevertheless, even the weakest inhibitory dose of penicillin renders the great majority of the spores non-viable. Spores studied in non-nutrient media were, however, little if at all affected, even by strong concentrations of penicillin, so that penicillin acts upon feeding bacteria and its action begins directly the bacteria begin to feed. Prof. Gardner concludes that the action of penicillin on sensitive species has little or no connexion with multiplication or division, although penicillin did cause abnormal divisions in sensitive $S$. typhi (long waists, spindle-shaped swellings, etc.). The majority of Staphylococcus aureus and Streptococcus pyogenes growing on agar are checked or killed before any visible growth or division has occurred. These conclusions, based upon direct microscopieal examination, must carry great weight. It would seem that what is now required for a better understanding of the mode of action of penicillin is more exact knowledge of the chemistry of penicillin and the correlation of this, by the methods now being used for the biochemical analysis of miero-organisms, with the enzyme systems and other metabolic features of the organisms concerned.

G. LaPage.

\section{RADIO WAVES FROM THE SUN}

A $\mathrm{S}$ the technique of radio-frequency measurement and application has progressed to successively higher frequencies, various investigators have from time to time turned their attention towards a demonstration of the interchangeability of thermal and radio methods of detecting electromagnetic waves. The use of normal radio receiving technique to detect thermal radiation from a hot body has always been an attractive line of experiment ; and for this purpose, the sun, in spite of its great distance away from the earth, would appear to offer considerable advantages as a source of radiation of the desired type. The practicability of the reception and measurement of so-called 'cosmic noise' on an ordinary radio receiver has been demonstrated in various investigations, notably those described during $1932-39$ by $K$. G. Jansky, who worked on frequencies of the order of 10-20 megacycles per second, and later (1940-44) by G. Reber, who described corresponding measurements made at frequencies of $160 \mathrm{Mc}$./s. In these cases, the 
investigators concluded that the radiation which they measured originated in interstellar space, and, from observations made of the direction of arrival, it was considered that the source of this cosmic noise was closely associated with the Milky Way. Both Jansky and Reber made attempts to observe any 'noise' radiation received from the sun, but although recently (Astrophys. J., Nov. 1944) the latter investigator claims to have obtained definite evidence of this, the amount of such radiation received at the above frequencies is very small.

In a paper entitled "Microwave Radiation from the $\operatorname{Sun}^{\prime}$ (J. Franklin Inst., April 1945), G. C. Southworth describes some experiments conducted in the summer months of 1942 and 1943 in which a small but measurable amount of radiation from the sun was obtained with a conventional type of radio receiver operating on frequencies between 3,000 and $30,000 \mathrm{Mc} . / \mathrm{s}$. (wave-lengths between 1 and $10 \mathrm{~cm}$.).

Following earlier work by Wien, Lord Rayleigh and Jeans, a complete formula for the energy distribution in the spectrum of a black-body radiation was derived by Planck from quantum considerations. At high temperatures and long wave-lengths, a condition of particular interest in the case of radio waves from the sun, the Planck relation reduces to the Rayleigh-Jeans formula. Applying this formula to the calculation of the energy distribution in the solar spectrum gives a result which is in general accord with experimental measurements throughout a considerable part of the visible and infra-red portions of the spectrum. The experimental results now deseribed. by Southworth tend to show that the same formula may be extended at least as far as the centimetrewave portion of the radio spectrum.

These recent measurements were made at three wave lengths between 1 and $10 \mathrm{~cm}$. using an antenna within a parabolic reflector of high directivity and a suitable type of radio receiver. At the longest of the three wave-lengths the measurements of received power agreed to within about \pm 5 per cent of that calculated by the Rayleigh-Jeans formula. At the intermediate wave-length the mean measured value was about 12 per cent below that calculated; while at the shortest wave-length, the mean observed level was approximately one-ninth of that calculated from the above formula. In no case was any marked diurnal variation observed such as occurs at infra-red wave-lengths, but at sunrise and sunset the received power varied more or less proportionally to the area of the sun's disk above the horizon; this transition period was passed through more slowly as the wavelength was reduced.

A particularly interesting part of the work described in Southworth's paper consisted of some directional measurements made by adjusting the receiver centrally on the sun and observing the decrease in output as the sun's disk moved out of the aperture of the receiving mirror. The effective angle of the latter, as measured by the displacement for which the received energy from a local source decreased to half the maximum value, varied from $\pm 3^{\circ}$ for the longest to $\pm 0 \cdot 3^{\circ}$ for the shortest wave-length used in the experiments. In the measurements of solar radiation, the apparent aperture was much wider than this at the shortest wave-length, where it amounted to more than $0 \cdot 8^{\circ}$, a result which is ascribed to the fact that the sun is an apparent disk and not a point source of radiation. At the lowest frequency, the angular diameter of the sun is small compared with the aperture angle of the receiver, but at the highest radio frequency used in this investigation, these two angles are comparable with one another. It may be expected that, to a first degree of approximation, the measured solar pattern will have a breadth equal to the sum of the aperture angle and the angular diameter of the sun. The fact that the observed directional pattern is even wider than this sum may be explained on the assumption that the angle of arrival of the waves from the sun varies rather rapidly over a considerable range; so that the sun appears to the receiver as a shimmering body the apparent diameter of which is considerably larger than the true diameter. This increase in apparent diameter may also account in part for the discrepancy between the measured and calculated energy levels already noted.

While carrying out the above work, it was found that the noise appearing in the output of the receiver was sensibly less when the receiver was pointed at the open sky above the horizon than when pointed at nearby objects or when it was located inside a room. The magnitude of this horizon effect for the longest wave used was of the order of $10^{-19}$ watts per sq. $\mathrm{cm}$. for a band width of $1 \mathrm{Mc}$./sec. The corresponding energy measured from the sun was about five times as great. At the shortest wave-length used, this 'horizon' effect was barely measurable, while the energy received from the sun was about $5 \times 10^{-18}$ watts per sq. $\mathrm{cm}$. for the same band-width conditions. Numerous attempts were made to observe cosmic noise coming from the general region of the Milky Way with the view of locating more precisely the source of the noise reported on by Jansky and Reber, but these attempts have so far proved unsuccessful on the higher frequencies used by Southworth. This experience tends to support Reber's view that cosmic radiation varies inversely as the frequency.

Perhaps the most interesting result of all this work is the establishment experimentally of a close relationship between black-body radiation and ordinary radio waves. Receiving from a source some 93 million miles away, it has been shown that the earth's atmosphere is fairly transparent to wave-lengths of the order of a centimetre, although there is evidence of absorption and scattering at the shortest wavelength at which observations were made. The next stage in the programme of investigation contemplated will incorporate the best ideas of both the radio engineer and the designer of astronomical equipment with the hope that additional accuracy will bring out features so far overlooked. Such observations on the transmission of radio waves through the earth's atmosphere from an outside source provide a means of investigating the structure of the atmosphere not previously available.

\section{NEW BUILDINGS OF BELL TELEPHONE LABORATORIES}

A $S$ soon as war restrictions permit, the facilities of Bell Telephone Laboratories at Murray Hill, N.J., will be greatly augmented, according to an announcement by Dr. Oliver E. Buckley, president of the Laboratories. The proposed addition is approximately the same size as the initial buildings, which were opened in 1941 and cost more than $2,000,000$ dollars. The new building will extend the lines of the present one about five hundred feet in a 